\title{
Duodenal stump cancer after Billroth-II distal gastrectomy for gastric cancer
}

\author{
Junhyun LeE ${ }^{1}$, Kyungus LeE ${ }^{2}$, and Wook Kim ${ }^{1}$ \\ ${ }^{1}$ Department of Surgery, Holy Family Hospital, College of Medicine, The Catholic University of Korea, 2 Sosa Dong, Wonmi Gu, Bucheon, \\ Gyunggi 420-717, Korea \\ ${ }^{2}$ Department of Hospital Pathology, Holy Family Hospital, College of Medicine, The Catholic University of Korea, Bucheon, Gyunggi, \\ Korea
}

\begin{abstract}
Duodenal cancer is an uncommon neoplasm and it mostly arises from the periampullary area. However, metachronous or even recurrent cancer at the duodenal stump following Billroth II type distal gastrec tomy for gastric cancer is extremely rare and, to our knowledge, has not yet been reported. A 68-year-old man underwent Billroth II distal gastrectomy with D2 lymph node dissection for an advanced gastric cancer. At that time the tumor stage was T2bN3M0, with 44 of 78 retrieved lymph nodes showing metastasis. He was well without recurrence for 3 years; however, he visited our hospital because of the abrupt onset of dizziness and tarry stool. A polypoid tumor that bled easily when touched was found at the end of the afferent loop of the duodenal stump by gastrofiberscopic examination, and it was proven to be an adenocarcinoma by endoscopic biopsy. Fortunately, additional studies, including abdominal computed tomography and positron emission tomography-computed tomography, showed no other sites of recurrence. The patient underwent pancreaticoduodenectomy for local control of the recurrent tumor at the duodenal stump, and the pathologic findings, based on immunohistochemical staining, strongly suggested that the duodenal tumor was metachronous in nature rather than recurrent.
\end{abstract}

Key words Advanced gastric cancer · Recurrence $\cdot$ Metachronous cancer $\cdot$ Duodenal stump

\section{Introduction}

Recently, the identification of early gastric cancer (EGC) in Korea has been rapidly increasing by virtue of nationwide gastrofiberscopic examinations. However, many patients still suffer from advanced cancer and postoperative cancer recurrences. Even patients with

Offprint requests to: $\mathrm{W}$. Kim

Received: December 8, 2008 / Accepted: April 23, 2009
EGC have a risk of developing a second primary (metachronous) cancer, such as colorectal, lung, or liver cancer [1-3].

The duodenal stump after Billroth II (B-II) distal gastrectomy is a rare site for cancer recurrence unless a tumor-free margin has not been achieved. Moreover, to our knowledge, there are no previously published studies regarding the occurrence of recurrent or metachronous cancer at the duodenal stump. Here, we describe duodenal stump cancer that occurred after a B-II distal gastrectomy for advanced gastric cancer (AGC), particularly focusing on the difficulties associated with distinguishing recurrent gastric cancer from metachronous duodenal cancer.

\section{Case report}

A 68-year-old man who had undergone distal gastrectomy with B-II type gastrojejunostomy for AGC in June 2005 , presented with abrupt onset of dizziness and tarry stool in June 2008. The previously removed tumor was $8.5 \times 7.5 \mathrm{~cm}$ in size, and located in the posterior wall of the mid-body portion of the stomach, and there was no duodenal lesion on the gastrofiberscopic (GFS) examination that had been performed before the gastrectomy. Pathologic examination revealed that the gastric lesion was a poorly differentiated adenocarcinoma infiltrating the subserosa, accompanied by multiple lymph node metastases (48 of 77 retrieved nodes were metastatic); the duodenum was resected, using a linear stapler, about $2 \mathrm{~cm}$ below the pyloric ring. The proximal and distal resected margins were $1.5 \mathrm{~cm}$ and $8.0 \mathrm{~cm}$, respectively. Subsequently, six cycles of adjuvant chemotherapy, including 5-fluorouracil (FU), epirubicin, and cisplatinum, were performed without any problems. The patient was followed up every 6 months by tumor marker analysis, ultrasonography, and computed tomography (CT) and his last checkup had been in January 2008. During 
the follow-up period, there had been no evidence of recurrence.

On admission, his laboratory findings were unremarkable, except for low hemoglobin $(7.2 \mathrm{~g} / \mathrm{ml})$ and hematocrit $(23.4 \%)$, which was managed with the transfusion of 5 units of packed RBC. GFS examination demonstrated a polypoid mass at the duodenal stump that bled easily when touched (Fig. 1A). Abdominal CT scan also revealed a mass at the duodenal stump (Fig. 1B) that had not been detected on the previous CT performed 6 months earlier. A biopsy of the polypoid mass revealed moderately differentiated adenocarcinoma. After confirmation of the absence of any other metastatic lesions by positron emission tomography (PET)-CT (Fig. 1C), we decided to remove the tumor because the patient needed a transfusion due to bleeding and it was thought that a palliative operation might be helpful to correct his anemia. When exploration of the abdominal cavity was carried out in June 2008, there were no findings suggesting that the tumor was inoperable, with no evidence of peritoneal seeding or of any sites of recurrence except for the duodenal lesion. Therefore, we performed pancreaticoduodenectomy with curative intent; the specimen obtained was $3.0 \times 2.5 \times 3.5 \mathrm{~cm}$ in size, and the tumor had invaded the peripancreatic soft tissues (Fig. 1D). There were three lymph node metastases out of seven retrieved lymph nodes, with lymphatic and perineural invasion. Pathologic analysis was used to compare the previous gastric lesion with the duodenal mass obtained in the present operation. HematoxylinEosin (Fig. 2A, B) and immunohistochemical (Fig. 3A-D) staining strongly indicated that the obtained specimen represented a metachronous duodenal cancer rather than a recurrent one.
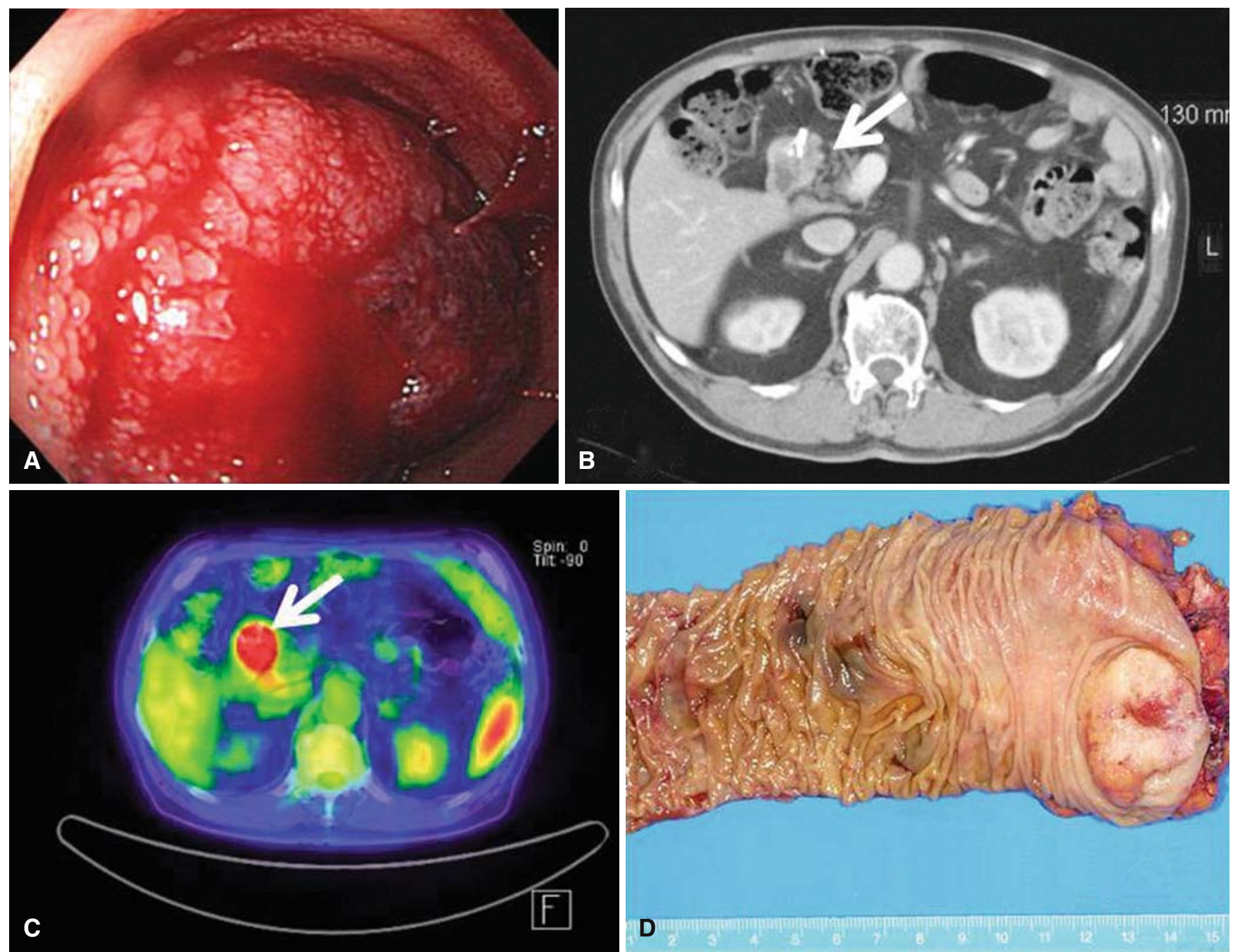

Fig. 1. A Gastrofiberscopic findings: polypoid mass with bleeding. B Abdominal computed tomography (CT) findings: arrow indicates lesion. C Positron emission tomography (PET)-CT detects hot uptake (arrow) in duodenal stump. D Resected specimen shows mass at the duodenal stump 

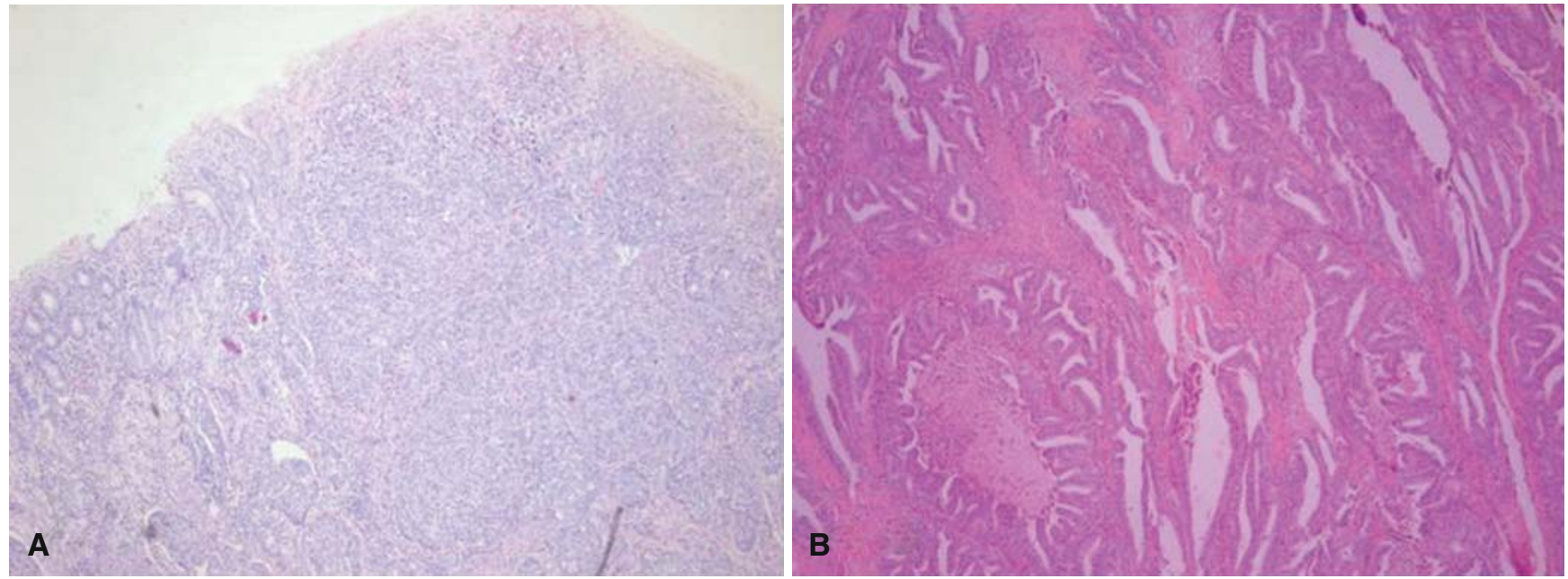

Fig. 2A,B. Pathologic findings. Tumor cells in the stomach and duodenum showed enlarged, pleomorphic, and hyperchromatic nuclei with prominent nucleoli. A The tumor that developed in the stomach was arranged in almost solid sheets. B Duodenal cancer showed well-formed elongated tubular structures without a solid portion. A and B H \& E, $\times 40$
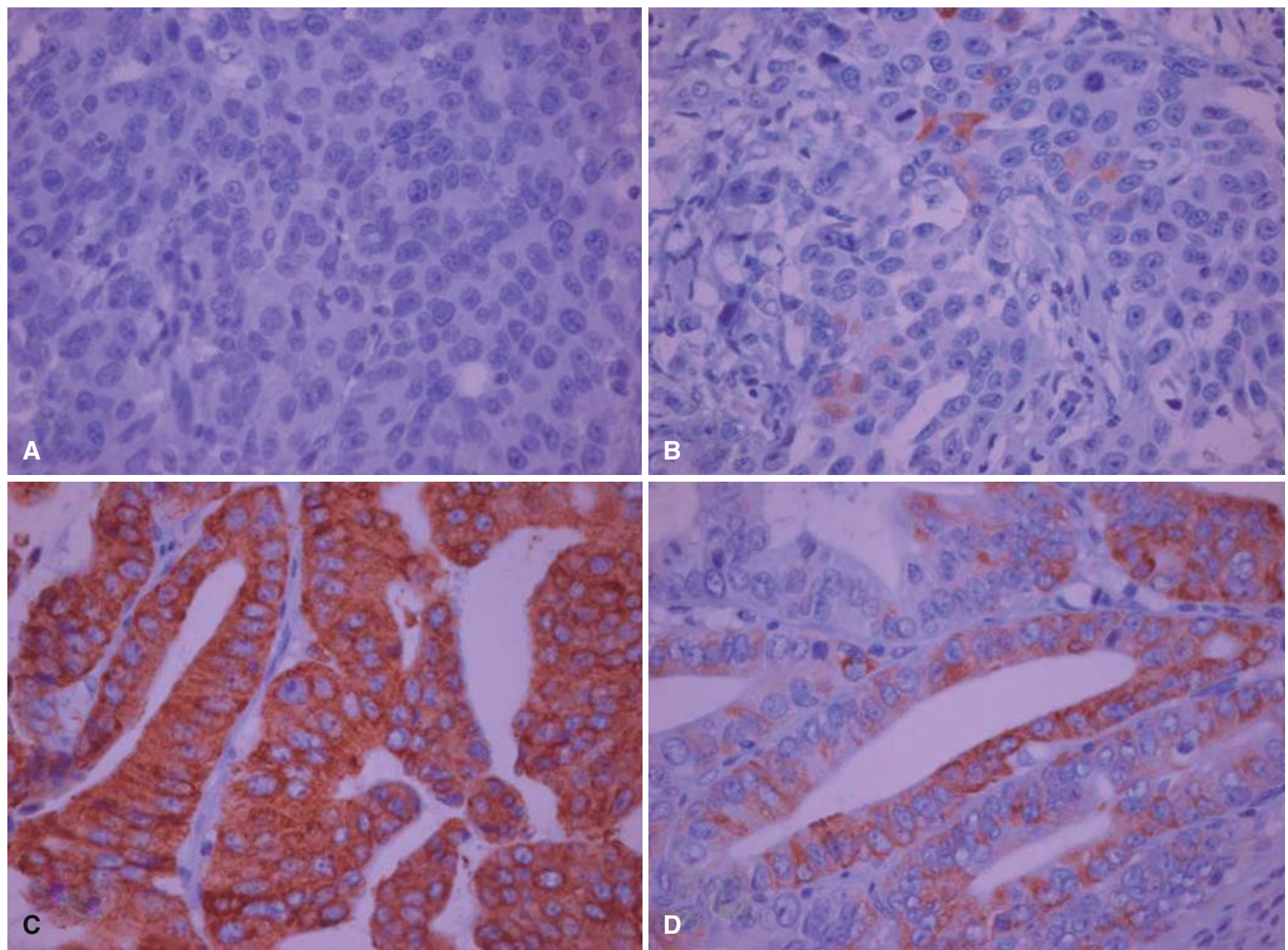

Fig. 3A-D. Immunohistochemical staining. A The gastric carcinoma was not stained by MUC5AC. B The gastric carcinoma was partly stained by only MUC6. C MUC5AC was diffusely positive in the duodenal carcinoma. D MUC6 was diffusely positive in the duodenal carcinoma. A, B, C, $\mathbf{D} \times 400$ 
The patient is free of recurrence now, 10 months after the second operation.

\section{Discussion}

Gastric cancer patients who have undergone gastrectomy have a risk of recurrence, especially those who have advanced disease. It is well known that peritoneal recurrence is most common, followed by hematogenous and lymph node recurrence [4]. Because of these risks, generally, modalities such as CT, ultrasound, and tumor marker (carcinoembryonic antigen) analysis have been used for follow up after gastrectomy. Recently, PET$\mathrm{CT}$, which has the advantage of being able to detect lesions that are not distinguished by CT, was introduced. PET-CT may be useful for gastric cancer patients in whom there is a clinical suspicion of recurrence [5]. However, PET-CT has limitations for the detection of recurrence if the histopathologic types of gastric cancer are mucinous and signet ring cell carcinoma, as well as poorly differentiated adenocarcinoma [6]. Therefore, there are still no sure tools for the detection of recurrence despite the availability of these diagnostic methods. In the present patient, sudden elevation of carbohydrate antigen 19-9 had been observed 30 months postoperatively. The patient had undergone abdominal CT with a suspicion of recurrence, but the CT did not reveal a lesion. Because the histopathologic type of gastric cancer that had been resected previously was poorly differentiated adenocarcinoma, we elected not to perform PET-CT, and we planned attentive followup measures. Six months after, a suspicious lesion was detected by both abdominal CT and PET-CT.

Our clinical opinion was that the duodenal stump mass in the present patient was a recurrent cancer resulting from invasion of the lymph nodes around the duodenal stump, which had been incompletely removed previously. However, pathologic examination with hematoxylin-eosin staining conflicted with our concept. So we carried out immunohistochemical staining including staining with MUC5AC, MUC6, and MUC2 to identify the origin of this tumor and compare it with the previous lesion. Gastrointestinal carcinomas are pathologically classified into four categories based on mucin type: mixed, gastric, intestinal, and unclassified, and prognosis of gastrointestinal carcinomas (or that) can be based on these subtypes [7]. In our immunohistochemical study, the tumor cells from both organs exhibited the gastric type. However, the staining patterns of the two tumors were different. The gastric cancer was partially stained by MUC6 alone. In contrast, the duodenal lesion displayed diffusely positive MUC5AC and MUC6 staining. These results were also suggestive of a metachronous tumor from the pathologic aspect.
Although the possibility of local recurrence at the duodenal stump was challenged by our pathologic findings, a review of the literature revealed that most duodenal cancers arise in the second portion of the duodenum and some occur in the third or fourth portion, but none occur in the first portion [8-10]. In addition to recurrence, metachronous cancer may develop in gastric cancer patients. Recently, some studies have reported that the risk of metachronous cancer associated with gastric cancer is elevated with increasing age, and this would negatively influence the prognosis of gastric cancer patients [1-3]. In published reports, the incidence of metachronous cancer occurring after gastric cancer is $1.4 \%-4.2 \%$, and it tends to occur more frequently in patients with EGC [1-3]. The colorectum is the most common site, followed by the lung and liver $[1,2]$. However, a significant correlation between metachronous duodenal cancer and gastric cancer has not yet been established, even though synchronous duodenal cancer associated with gastric cancer has been reported [11]. On consideration of these clinical features of duodenal cancer and the relationship with gastric cancer, we have not yet clarified the origin of the duodenal stump lesion in our patient, although the pathologic findings strongly suggested that the tumor was metachronous.

Recurrence is the most feared factor in patients with gastric cancer and, unfortunately, most recurrences are at multiple sites at the time of diagnosis [12]. The standard treatment for recurrence has not been well established, but, on the other hand, a few studies have demonstrated that, in cases of localized recurrence, complete resection, when achievable, may be beneficial to the patient's survival $[13,14]$. Because the lesion in our patient was regarded as a localized recurrence, we performed the operation and patient has not shown any signs of recurrence as yet.

In summary, the unusual location of the duodenal stump, including the surrounding lymph nodes, makes it difficult to differentiate metachronous duodenal cancer from recurrence in this region. However, regardless of its origin, the rare case presented herein was successfully managed by pancreaticoduodenectomy. Routine follow up with attention may be helpful for early detection and treatment in such types of cases.

\section{References}

1. Eom BW, Lee HJ, Yoo MW, Cho JJ, Kim WH, Yang HK, et al. Synchronous and metachronous cancers in patients with gastric cancer. J Surg Oncol 2008;98:106-10.

2. Ikeda Y, Saku M. Kawanaka H, Nonaka M, Yoshida K. Features of second primary cancer in patients with gastric cancer. Oncology 2003;65:113-7. 
3. Dinis-Ribeiro M, Lomba-Viana H, Silva R, Moreira-Dias L, Lomba-Viana R. Associated primary tumors in patients with gastric cancer. J Clin Gastroenterol 2002;34:533-5.

4. Saito H, Tsujitani S, Kondo A, Ikeguchi M, Maeta M, Kaibara N. Expression of vascular endothelial growth factor correlates with hematogenous recurrence in gastric carcinoma. Surgery 1999; 125:195-201.

5. Jadvar H, Tatlidil R, Garcia AA, Conti PS. Evaluation of recurrent gastric malignancy with [F-18]-FDG positron emission tomography. Clin Radiol 2003;58:215-21.

6. Lim JS, Yun MJ, Kim MJ, Hyung WJ, Park MS, Choi JY, et al. $\mathrm{CT}$ and PET in the stomach cancer: preoperative staging and monitoring of response to therapy. Radiographics 2006;26: $143-56$.

7. Lee HS, Lee HK, Kim HS, Yang HK, Kim YI, Kim WH. MUC1, MUC2, MUC5AC, and MUC6 expressions in gastric carcinomas: their roles as prognostic indicators. Cancer 2001;92:1427-34.

8. Iovine VM, Tsangaris N. Primary carcinoma on the duodenum. Am Surg 1961;27:744-50.
9. Ryder NM, Ko CY, Hines OJ, Gloor B, Reber HA. Primary duodenal adenocarcinoma. Arch Surg 2000;135:1070-5.

10. Scott-Coombes DM, Williamson RCN. Surgical treatment of primary duodenal carcinoma: a personal series. Br J Surg 1994; 81:1472-4.

11. Onoue S, Katoh T, Chigira H, Matsuo K, Suzuki M, Shibata Y, et al. Synchronous multiple primary cancers of the stomach and duodenum in aged patients: report of two cases. Surg Today 2000;30:735-8.

12. Yoo $\mathrm{CH}$, Noh SH, Shin DW. Recurrence following curative resection for gastric carcinoma. Br J Surg 2000;87:236-42.

13. Song KY, Park SM, Kim SM, Park CH. The role of surgery in the treatment of recurrent gastric cancer. Am J Surg 2008;196: 19-22.

14. Shchepotin I, Evans SR, Shabahang M, Cherny M, Buras MM, Zadorozhny A. Radical treatment of locally recurrent gastric cancer. Am Surg 1995;6:371-6. 